\title{
AN OPTIMAL MASS TRANSPORT APPROACH FOR LIMITS OF EIGENVALUE PROBLEMS FOR THE FRACTIONAL $p$-LAPLACIAN.
}

\author{
L. M. DEL PEZZO, J. D. ROSSI, N. SAINTIER, AND A. SALORT
}

\begin{abstract}
We find interpretation using optimal mass transport theory for eigenvalue problems obtained as limits of the eigenvalue problems for the fractional $p$-Laplacian operators as $p \rightarrow+\infty$. We deal both with Dirichlet and Neumann boundary conditions.
\end{abstract}

\section{INTRODUCTION}

Our main goal in this paper is to use tools from mass transport theory to study eigenvalue problems that are obtained taking limits as $p \rightarrow+\infty$ in eigenvalue problems that involve fractional spaces $W^{s, p}$ (with $0<s<1$ and $1<p \leq+\infty)$. We deal both with Dirichlet and Neumann boundary conditions.

Along this paper we take $U$ a smooth bounded domain in $\mathbb{R}^{n}, 1<p<+\infty$ and $0<s<1$. We also fix $d(\cdot, \cdot)$ a distance in $\mathbb{R}^{n}$ equivalent to the Euclidean one.

Let $\lambda_{s, p}^{D}$ be the first eigenvalue of the fractional $p$-Laplacian of order $s$ in $U$ with Dirichlet boundary conditions, that is, let us consider

$$
\lambda_{s, p}^{D}:=\inf \left\{[u]_{s, p}^{p}: u \in \widetilde{W}^{s, p}(U), \int_{U}|u|^{p} d x=1\right\}
$$

where

$$
[u]_{s, p}^{p}:=\int_{\mathbb{R}^{n}} \int_{\mathbb{R}^{n}} \frac{|u(x)-u(y)|^{p}}{|x-y|^{n+s p}} d x d y
$$

is the semi-norm of $W^{s, p}\left(\mathbb{R}^{n}\right)$ and

$$
\widetilde{W}^{s, p}(U):=\left\{u \in W^{s, p}\left(\mathbb{R}^{n}\right): u=0 \text { in } \mathbb{R}^{n} \backslash U\right\} .
$$

For this problem Lindgren and Lindqvist in [18] proved that

$$
\Lambda_{s, \infty}^{D}:=\lim _{p \rightarrow+\infty}\left(\lambda_{s, p}^{D}\right)^{1 / p}=\frac{1}{R^{s}}
$$

Date: January 16, 2015.

Key words and phrases. Fractional $p$-Laplacian, eigenvalues, mass transport.

2010 Mathematics Subject Classification: 35J92, 35P30, 45 C05. 
where

$$
R:=\max _{x \in \bar{U}} \operatorname{dist}(x, \partial U)=\max _{x \in \bar{U}} \min _{y \in \partial U}|x-y| .
$$

Moreover, via a subsequence, the eigenfunctions $u_{p}$ suitable normalized (a minimizer for $\lambda_{s, p}^{D}$ ) converge uniformly to a minimizer for $\Lambda_{s, \infty}^{D}$.

Our first purpose in this work is to relate $\Lambda_{s, \infty}^{D}$ to an optimal mass transport problem with cost function $c(x, y)=|x-y|^{s}$. We prove the following result:

Theorem 1.1. There holds that

$$
\frac{1}{\Lambda_{s, \infty}^{D}}=\sup _{\mu \in P(\bar{U})} W_{s}(\mu, P(\partial U))
$$

where

$$
W_{s}(\mu, \nu):=\inf _{\pi} \int_{U} \int_{U}|x-y|^{s} d \pi(x, y)
$$

Here $P(A)$ is the set of probability measures on $A$ and $\pi \in P(\bar{U} \times \bar{U})$ is a measure with marginals $\mu$ and $\nu$.

Note that $W_{s}(\mu, \nu)$ is the total cost when we have to transport the measure $\mu$ onto $\nu$ using as cost for transporting one unit of mass from position $x$ to position $y$ the Euclidean distance to the power $s$, that is $|x-y|^{s}$. We refer to [20] and to Section 2 for precise definitions, notations and properties of optimal mass transport theory. Hence, our result says that the eigenvalue $\Lambda_{s, \infty}^{D}$ is related to the problem of finding a probability measure supported inside $U, \mu$, that is far (in terms of the transport cost) from the set of probability measures supported on the boundary, $\partial U$. One easy solution to this problem is the following: take $B_{R}\left(x_{0}\right)$ a ball with maximum radius $R$ inside $U$ and let $y_{0} \in \partial U \cap \partial B_{R}\left(x_{0}\right)$ (there exists such $y_{0}$ due to the maximality of $R)$. Then, $\mu=\delta_{x_{0}}\left(\right.$ with $\left.\nu=\delta_{y_{0}}\right)$ solves $\sup _{\mu \in P(\bar{U})} W_{s}(\mu, P(\partial U))$. Observe that from Theorem 1.1 we can recover that $\Lambda_{s, \infty}^{D}=1 / R^{s}$.

Now, let us turn our attention to the case of the first nontrivial eigenvalue for Neumann boundary conditions, i.e., let us consider

$$
\lambda_{s, p}^{N}:=\inf \left\{\llbracket u \rrbracket_{s, p}^{p}: u \in \mathcal{C}\right\}
$$

where

$$
\llbracket u \rrbracket_{s, p}^{p}:=\int_{U} \int_{U} \frac{|u(x)-u(y)|^{p}}{d(x, y)^{n+s p}} d x d y
$$

and

$$
\mathcal{C}:=\left\{u \in W^{s, p}(U):\|u\|_{L^{p}(U)}=1, \int_{U}|u|^{p-2} u d x=0\right\} .
$$

For this problem, in the case $d(x, y)=|x-y|$, Del Pezzo and Salort in [8] proved that

$$
\Lambda_{s, \infty}^{N}:=\lim _{p \rightarrow+\infty}\left(\lambda_{s, p}^{N}\right)^{1 / p}=\frac{2}{(\operatorname{diam}(U))^{s}}
$$


where $\operatorname{diam}(U)$ is the extrinsic diameter, i.e.

$$
\operatorname{diam}(U):=\max _{x, y \in \bar{U}}|x-y| .
$$

Their proof actually extends to the case in which we consider $\llbracket u \rrbracket_{s, p}^{p}$ with $d(x, y)$ any distance as above (for instance, for the geodesic distance in $U$ ). In this case, it holds that

$$
\Lambda_{s, \infty}^{N}=\lim _{p \rightarrow+\infty}\left(\lambda_{s, p}^{N}\right)^{1 / p}=\frac{2}{\left(\operatorname{diam}_{d}(U)\right)^{s}}
$$

where $\operatorname{diam}_{d}(U)$ is the diameter of $U$ according to $d$, that is

$$
\operatorname{diam}_{d}(U)=\max _{x, y \in \bar{U}} d(x, y) .
$$

Moreover, as happens for the Dirichlet problem, via a subsequence the normalized eigenfunctions $u_{p}$ (a minimizer for $\lambda_{s, p}^{N}$ ) converge uniformly to a minimizer for $\Lambda_{s, \infty}^{N}$.

In order to introduce the mass transport interpretation we need the following notations. We denote by $M(\bar{U})$ the space of finite Borel measures over $\bar{U}$. Given $\sigma \in M(\bar{U})$, we denote its positive and negative part by $\sigma^{+}$ and $\sigma^{-}$so that $\sigma=\sigma^{+}-\sigma^{-}$, and $|\sigma|=\sigma^{+}+\sigma^{-}$. Then we have,

Theorem 1.2. There holds

$$
\frac{2}{\Lambda_{s, \infty}^{N}}=\max \left\{W_{s}\left(\sigma^{+}, \sigma^{-}\right): \sigma \in M(\bar{U}), \sigma^{+}(\bar{U})=\sigma^{-}(\bar{U})=1\right\}
$$

where $W_{s}$ is as in Theorem 1.1.

Here we relate $\Lambda_{s, \infty}^{N}$ to the problem of finding two probability measures, $\sigma^{+}$and $\sigma^{-}$, supported in $\bar{U}$, such that the cost of transporting one into the other is maximized. To obtain a solution to this problem one can argue as follows: take two points $x_{0}$ and $y_{0}$ in $\bar{U}$ that realize the diameter, that is, we have $d\left(x_{0}, y_{0}\right)=\operatorname{diam}_{d}(U)$. Then take $\sigma^{+}=\delta_{x_{0}}$ and $\sigma^{-}=\delta_{y_{0}}$ as a solution to $\max \left\{W_{s}\left(\sigma^{+}, \sigma^{-}\right): \sigma \in M(\bar{U}), \sigma^{+}(\bar{U})=\sigma^{-}(\bar{U})=1\right\}$. Note that we can recover (1.2) from Theorem 1.2.

A different concept of Neumann boundary condition for fractional operators was recently introduced in [9]. More precisely, for $(-\Delta)_{p}^{s}$ the fractional $p$-Laplacian given by

$$
(-\Delta)_{p}^{s} u(x)=\mathrm{P} . \mathrm{V} \cdot \int_{\mathbb{R}^{n}} \frac{|u(x)-u(y)|^{p-2}(u(x)-u(y))}{d(x, y)^{n+s p}} d y
$$

(the symbol P.V. stands for the principal value of the integral), we consider the following non-local non-linear fractional normal derivative

$$
\mathcal{N}_{s, p} u(x)=\int_{U} \frac{|u(x)-u(y)|^{p-2}(u(x)-u(y))}{d(x, y)^{n+s p}} d y \quad x \in \mathbb{R}^{n} \backslash \bar{U} .
$$


Associated with this operator, we consider the following eigenvalue problems

$$
\begin{cases}(-\Delta)_{p}^{s} u=\lambda|u|^{p-2} u & \text { in } U \\ \mathcal{N}_{s, p} u=0 & \text { in } \mathbb{R}^{n} \backslash \bar{U} .\end{cases}
$$

Before stating our main result concerning these problems, we need to introduce some notations. Let $\mathcal{W}^{s, p}(U)$ be the set of measurable functions with finite

$$
\|u\|_{\mathcal{W}^{s, p}(U)}^{p}:=\|u\|_{L^{p}(U)}^{p}+\mathcal{H}_{s, p}(u)
$$

where

$$
\mathcal{H}_{s, p}(u):=\iint_{\mathbb{R}^{2 n} \backslash\left(U^{c}\right)^{2}} \frac{|u(x)-u(y)|^{p}}{d(x, y)^{n+p s}} d x d y,
$$

and $\left(U^{c}\right)^{2}=U^{c} \times U^{c}$. Let us also introduce

$$
\mathcal{H}_{s, \infty}(u):=\sup \left\{\frac{|u(x)-u(y)|}{d(x, y)^{s}}:(x, y) \in \mathbb{R}^{2 n} \backslash\left(U^{c}\right)^{2}\right\} .
$$

Then, for (1.4) we have the following result.

Theorem 1.3. The first non-zero eigenvalue of (1.4) is given by

$$
\lambda_{s, p}=\inf \left\{\frac{\mathcal{H}_{s, p}(v)}{2\|v\|_{L^{p}(U)}^{p}}: v \in \mathcal{W}^{s, p}(U) \backslash\{0\}, \int_{U}|v|^{p-2} v d x=0\right\} .
$$

Concerning the limit as $p \rightarrow+\infty$ of these eigenvalues we have

$$
\lim _{p \rightarrow+\infty}\left(\lambda_{s, p}\right)^{1 / p}=\frac{2}{\left(\operatorname{diam}_{d}(U)\right)^{s}}=\Lambda_{s, \infty}:=\inf \left\{\frac{\mathcal{H}_{s, \infty}(u)}{\|u\|_{L^{\infty}(U)}}: u \in \mathcal{A}\right\},
$$

where

$$
\mathcal{A}:=\left\{v \in \mathcal{W}^{s, \infty}(U) \backslash\{0\}: \sup _{x \in U} u(x)+\inf _{x \in U} u(x)=0\right\} .
$$

Moreover, if $u_{p}$ is a minimizer of $\lambda_{s, p}$ normalized by $\left\|u_{p}\right\|_{L^{p}(U)}=1$, then, up to a subsequence, $u_{p}$ converges in $C(\bar{U})$ to some minimizer $u_{\infty} \in W^{s, \infty}(U)$ of $\Lambda_{s, \infty}^{N}$.

Note that, since the limit of $\left(\lambda_{s, p}\right)^{1 / p}, \Lambda_{s, \infty}$, coincides with $\Lambda_{s, \infty}^{N}$ (given in (1.2)), we get the same interpretation in terms of optimal mass transportation given in Theorem 1.2.

To end this introduction, let us briefly comment on previous results. The limit as $p \rightarrow+\infty$ of the first eigenvalue $\lambda_{p}^{D}$ of the usual local $p$-Laplacian with Dirichlet boundary condition was studied in [15, 16], (see also [3] for an anisotropic version). In those papers the authors prove that

$$
\lambda_{\infty}^{D}:=\lim _{p \rightarrow+\infty}\left(\lambda_{p}^{D}\right)^{1 / p}=\inf \left\{\frac{\|\nabla v\|_{L^{\infty}(U)}}{\|v\|_{L^{\infty}(U)}}: v \in W_{0}^{1, \infty}(\Omega)\right\}=\frac{1}{R},
$$

where $R$ is, as before, the largest possible radius of a ball contained in $U$. In addition, the authors show the existence of extremals, i.e. functions where 
the above infimum is attained. These extremals can be constructed taking the limit as $p \rightarrow+\infty$ in the eigenfunctions of the $p$-Laplacian eigenvalue problems (see [15]) and are viscosity solutions of the following eigenvalue problem (called the infinity eigenvalue problem in the literature):

$$
\begin{cases}\min \left\{|D u|-\lambda_{\infty}^{D} u, \Delta_{\infty} u\right\}=0 & \text { in } U \\ u=0 & \text { on } \partial U\end{cases}
$$

The limit operator $\Delta_{\infty}$ that appears here is the $\infty$-Laplacian given by $\Delta_{\infty} u=-\left\langle D^{2} u D u, D u\right\rangle$. Remark that solutions to $\Delta_{p} v_{p}=0$ with a Dirichlet data $v_{p}=f$ on $\partial U$ converge as $p \rightarrow+\infty$ to the viscosity solution to $\Delta_{\infty} v=0$ with $v=f$ on $\partial U$, see $[2,4,6]$. This operator appears naturally when one considers absolutely minimizing Lipschitz extensions in $U$ of a boundary data $f$, see $[1,2,13]$.

Recently in [5], the authors relate $\lambda_{\infty}^{D}$ with the Monge-Kantorovich distance $W_{1}$. Recall that the Monge-Kantorovich distance $W_{1}(\mu, \nu)$ between two probability measures $\mu$ and $\nu$ over $\bar{U}$ is defined by

$$
W_{1}(\mu, \nu):=\max \left\{\int_{U} v(d \mu-d \nu): v \in W^{1, \infty}(U),\|\nabla v\|_{L^{\infty}(U)} \leq 1\right\} .
$$

It was proved in [5] that

$$
\frac{1}{\lambda_{\infty}^{D}}=\sup _{\mu \in P(U)} W_{1}(\mu, P(\partial U)) .
$$

Notice that this result is the analogous to Theorem 1.1 in the local case.

For the Neumann problem for the local $p$-Laplacian we refer to $[10,19]$ where the authors prove the local analogous to Theorem 1.2. In this local case the distance that appears in the limit is the geodesic distance inside $U$. This is, in contrast with the non-local case studied here, where we can consider any distance $d$ equivalent to the Euclidean one, see (1.1).

For limits as $p \rightarrow+\infty$ in non-local $p$-Laplacian problems and its relation with optimal mass transport we refer to [14]. Eigenvalue problems were not considered there.

The case of a Steklov boundary condition has also been investigated recently. Indeed, the authors in [12] (see also [17] for a slightly different problem) studied the behavior as $p \rightarrow+\infty$ of the so-called variational eigenvalues $\lambda_{k, p}^{S}, k \geq 1$, of the $p$-Laplacian with a Steklov boundary condition. In particular they proved that

$$
\lim _{p \rightarrow+\infty}\left(\lambda_{1, p}^{S}\right)^{1 / p}=1 \quad \text { and } \quad \lambda_{2, \infty}^{S}:=\lim _{p \rightarrow+\infty}\left(\lambda_{2, p}^{S}\right)^{1 / p}=\frac{2}{\operatorname{diam}(U)},
$$

and also identify the limit variational problem defining $\lambda_{2, \infty}^{S}$.

The paper is organized as follows: in Section 2 we collect some preliminary results concerning optimal mass transport with cost $d(x, y)^{s}$, in particular, we provide a statement of the Kantorovich duality result that will be used in 
the proofs of our results; in Section 3 we deal with the Dirichlet problem and prove Theorem 1.1; in Section 4 we study the Neumann case (Theorem 1.2). Finally, in Section 5 we deal with problem (1.4) and we prove Theorem 1.3.

\section{Kantorovich DUALity For the COST $c(x, y)=d(x, y)^{s}$}

In this section we follow [20]. We first recall the definition of $c$-concavity and $c$-transform.

Definition 2.1 ([20, Definitions 5.2 and 5.7]). Let $X, Y$ be two sets and $c: X \times Y \rightarrow \mathbb{R} \cup\{+\infty\}$. A function $\psi: X \rightarrow \mathbb{R} \cup\{+\infty\}$ is said to be c-convex if $\psi \not \equiv+\infty$ and there exists $\zeta: Y \rightarrow \mathbb{R} \cup\{ \pm \infty\}$ such that

$$
\psi(x)=\sup _{y \in Y} \zeta(y)-c(x, y) \quad \text { for all } x \in X .
$$

Its c-transform is the function $\psi^{c}$ defined by

$$
\psi^{c}(y)=\inf _{x \in X} \psi(x)+c(x, y) \quad \text { for all } y \in Y .
$$

A function $\phi: Y \rightarrow \mathbb{R} \cup\{-\infty\}$ is c-concave if $\phi \not \equiv-\infty$ and $\phi=\psi^{c}$ for some function $\psi: X \rightarrow \mathbb{R} \cup\{ \pm \infty\}$. Then its c-transform $\phi^{c}$ is

$$
\phi^{c}(x)=\sup _{y \in Y} \phi(y)-c(x, y) \quad \text { for all } x \in X .
$$

Notice that $\psi$ is $c$-convex iff $\psi=-\zeta^{c}$ for some function $\zeta$. Moreover,

Proposition 2.2 ([20, Proposition 5.8]). For any $\psi, \psi^{c}=\psi^{c c c}$ and $\psi$ is c-convex iff $\psi=\psi^{\text {cc }}$.

In the case where the cost function is $c(x, y)=d(x, y)^{s}$ for some $s \in \mathbb{R}^{+}$, we have the following characterization of $c$-convex function.

Lemma 2.3. Let $c(x, y)=d(x, y)^{s}$ and $X=Y=\bar{U}$. Then a function $\phi: \bar{U} \rightarrow \mathbb{R} \cup\{+\infty\}$ is c-concave iff

$$
|\phi(x)-\phi(\tilde{x})| \leq d(x, \tilde{x})^{s} \quad \text { for all } x, \tilde{x} \in \bar{U} .
$$

Moreover in that case, $\phi^{c}=\phi$.

Proof. Notice that

$$
\phi^{c}(x)=\sup _{y \in \bar{U}} \phi(y)-d(x, y)^{s} \geq \phi(x)
$$

and that the opposite inequality holds if (2.1) holds. Hence if $\phi$ satisfies (2.1) then $\phi^{c}=\phi$. So that in particular $\phi$ is $c$-concave.

Reciprocally, assume that $\phi$ is $c$-concave so that

$$
\phi(x)=\psi^{c}(x)=\inf _{y \in \bar{U}} \psi(y)+d(x, y)^{s}
$$


for some function $\psi$. Since $s \in(0,1)$, we have $d(x, y)^{s} \leq d(x, \tilde{x})^{s}+d(y, \tilde{x})^{s}$ for any $x, \tilde{x}, y \in \bar{U}$. It follows that

$$
\phi(x) \leq \inf _{y \in \bar{U}} \psi(y)+d(y, \tilde{x})^{s}+d(x, \tilde{x})^{s}=\phi(\tilde{x})+d(x, \tilde{x})^{s},
$$

i.e. $\phi(x)-\phi(\tilde{x}) \leq d(x, \tilde{x})^{s}$. The opposite inequality holds as well by switching $x$ and $\tilde{x}$. Thus (2.1) holds.

We recall the following result, see [20, Theorem 5.10].

Theorem 2.4. Let $(X, \mu)$ and $(Y, \nu)$ be two Polish spaces (i.e. metric complete separable) and let $c: X \times Y \rightarrow \mathbb{R} \cup\{+\infty\}$ be a lower semicontinuous function such that

$$
c(x, y) \geq a(x)+b(y) \quad \text { for all }(x, y) \in X \times Y
$$

for some real-valued upper semicontinuous functions $a \in L^{1}(\mu)$ and $b \in$ $L^{1}(\nu)$. Then

$$
W_{c}(\mu, \nu):=\inf _{\pi \in \Pi(\mu, \nu)} \int_{X \times Y} c(x, y) d \pi(x, y)=\sup _{\phi \in L^{1}(\nu)} \int_{Y} \phi d \nu-\int_{X} \phi^{c} d \mu,
$$

and in the above sup, one might as well impose $\phi$ to be c-concave. Moreover if $c$ is real-valued, $W_{c}(\mu, \nu)<\infty$ and

$$
c(x, y) \leq c_{X}(x)+c_{Y}(y) \quad \text { for all }(x, y) \in X \times Y
$$

for some $c_{X} \in L^{1}(\nu)$ and $c_{Y} \in L^{1}(\mu)$, then the above sup is a max.

In the particular case $c(x, y)=d(x, y)^{s}, X=Y=\bar{U}$ with $U$ bounded, we obtain in view of Lemma 2.3 the following result.

Theorem 2.5. For any $\mu, \nu \in P(\bar{U})$,

$$
\begin{aligned}
\min _{\pi \in \Pi(\mu, \nu)} \int_{\bar{U} \times \bar{U}} d(x, y)^{s} d \pi(x, y) & =\max _{\phi \in L^{1}(\nu) c \text {-concave }} \int_{\bar{U}} \phi d \nu-\int_{\bar{U}} \phi^{c} d \mu \\
& =\max _{|\phi(x)-\phi(y)| \leq d(x, y)^{s}} \int_{\bar{U}} \phi d \nu-\int_{\bar{U}} \phi d \mu .
\end{aligned}
$$

\section{The Dirichlet CASe}

In this section, we borrow ideas from [5]. Let us consider

$$
G_{p}, G_{\infty}: C(\bar{U}) \times M(\bar{U}) \rightarrow \mathbb{R} \cup\{+\infty\}
$$

the functionals given by

$$
G_{p}(v, \sigma)= \begin{cases}-\int_{U} v \sigma d x, & \text { if } \sigma \in L^{p^{\prime}}(U),\|\sigma\|_{L^{p^{\prime}}(U)} \leq 1 \\ +\infty & \text { and } v \in \widetilde{W}^{s, p}(U),[v]_{s, p} \leq\left(\lambda_{s, p}^{D}\right)^{1 / p} \\ & \text { otherwise, }\end{cases}
$$


and

$$
G_{\infty}(v, \sigma)= \begin{cases}-\int_{U} v d \sigma, & \text { if } \sigma \in M(\bar{U}),|\sigma|(U) \leq 1, \\ & \text { and } v \in \widetilde{W}^{s, \infty}(U),|v(x)-v(y)| \leq \Lambda_{s, \infty}^{D}|x-y|^{s} \\ +\infty & \text { otherwise. }\end{cases}
$$

In the space $M(\bar{U})$, we consider the weak convergence of measures, and in the space $C(\bar{U})$ the uniform convergence.

First, we have that $G_{\infty}$ is the limit as $p \rightarrow+\infty$ of $G_{p}$ in the $\Gamma$-limit sense (we refer to [7] for the definition of $\Gamma$-convergence).

Lemma 3.1. The functionals $G_{p} \Gamma$-converge as $p \rightarrow+\infty$ to $G_{\infty}$.

Proof. It follows as in [5].

Now, we let $f_{p}: \mathbb{R}^{n} \rightarrow \mathbb{R}$ defined as

$$
f_{p}(x):=\left(u_{p}(x)\right)^{p-1}
$$

where $u_{p}$ is a nonnegative eigenfunction associated to $\lambda_{s, p}^{D}(U)$ such that $\left\|u_{p}\right\|_{L^{p}(U)}=1$. When we consider $f_{p}$ as an element of $M(\bar{U})$ together with $u_{p}$ we obtain a minimizer for $G_{p}$. The proof of this fact is immediate.

Lemma 3.2. The pair $\left(f_{p}, u_{p}\right)$ minimizes $G_{p}$ in $C(\bar{U}) \times M(\bar{U})$ with

$$
G_{p}\left(f_{p}, u_{p}\right)=-1 \text {. }
$$

Now, let us show that we can extract a subsequence $p_{n} \rightarrow+\infty$ such that $f_{p}$ and $u_{p}$ converge.

Lemma 3.3. There exists a sequence $p_{n} \rightarrow+\infty$ such that

$$
u_{p_{n}} \rightarrow u_{\infty}
$$

uniformly in $\mathbb{R}^{n}$. This limit $u_{\infty}$ verifies

$$
\left|u_{\infty}(x)-u_{\infty}(y)\right| \leq \Lambda_{s, \infty}^{D}|x-y|^{s}, \quad x, y \in \mathbb{R}^{n} .
$$

Moreover, we have

$$
f_{p_{n}} \stackrel{*}{\rightarrow} f_{\infty}
$$

weakly-* in $M(\bar{U})$ and $f_{\infty}$ is a nonnegative measure that verifies $f_{\infty}(\bar{U}) \leq 1$.

Proof. The convergence of $u_{p}$, via a subsequence, is contained in [18]. Concerning $f_{p_{n}}$ the conclusion follows from the inequality

$$
\int_{U} f_{p} d x \leq\left(\int_{U}\left(u_{p}\right)^{p} d x\right)^{\frac{p-1}{p}}|U|^{1 / p}=|U|^{1 / p},
$$

that implies that $f_{p}$ is bounded in $M(\bar{U})$ and hence we can extract a sequence $p_{n} \rightarrow+\infty$ such that $f_{p_{n}} \stackrel{*}{\rightarrow} f_{\infty}$ weakly-* in $M(\bar{U})$. That the limit $f_{\infty}$ is a nonnegative measure that verifies $f_{\infty}(\bar{U}) \leq 1$ also follows from (3.1). 
From the main property of $\Gamma$-convergence we obtain the following corollary.

Corollary 3.4. The pair $\left(f_{\infty}, u_{\infty}\right)$ minimizes $G_{\infty}$ with

$$
G_{\infty}\left(f_{\infty}, u_{\infty}\right)=-1
$$

Now we are ready to prove Theorem 1.1.

Proof of Theorem 1.1. As $\left(f_{\infty}, u_{\infty}\right)$ minimizes $G_{\infty}$ we obtain that

$$
\left(f_{\infty}, \frac{u_{\infty}}{\Lambda_{\infty, s}^{D}}\right)
$$

minimizes

$$
-\int_{U} v d \sigma
$$

with $(v, \sigma)$ belonging to

$$
A:=\left\{(v, \sigma) \in \widetilde{W}^{s, \infty}(U) \times M(\bar{U}):|\sigma|(U) \leq 1,|v(x)-v(y)| \leq d(x, y)^{s}\right\} .
$$

Then

$$
\begin{aligned}
\frac{1}{\Lambda_{s, \infty}^{D}} & =\frac{1}{\Lambda_{s, \infty}^{D}} \int_{U} u_{\infty} d f_{\infty} \\
& =\max _{(v, \sigma) \in A} \int_{U} v d \sigma \\
& =\max _{\mu \in P(\bar{U})} \max _{w(x)-w(y) \mid \leq d(x, y)^{s}} \int_{U} w d \mu \\
& =\max _{\mu \in P(\bar{U})} W_{s}(\mu, P(\partial U)),
\end{aligned}
$$

as we wanted to show.

\section{The Neumann CASE}

Again, we follow ideas from [5], see also [19]. Let $u_{p}$ be an extremal for $\lambda_{p, s}^{N}$ (that is, a minimizer for (1.1)) normalized by $\left\|u_{p}\right\|_{L^{p}(U)}=1$. Then $f_{p}:=\left|u_{p}\right|^{p-2} u_{p} \in L^{p^{\prime}}(U)$ (where $p^{\prime}=\frac{p}{p-1}$ ) satisfies

$$
\left\|f_{p}\right\|_{L^{p^{\prime}(U)}}=1 \quad \text { and } \quad \int_{U} f_{p} d x=0 .
$$

The first step consists in extracting from $\left\{f_{p}\right\}_{p>1}$ a subsequence converging weakly to some measure $f_{\infty} \in M(\bar{U})$, the weak convergence meaning here that

$$
\lim _{p \rightarrow+\infty} \int_{\bar{U}} \phi f_{p} d x=\int_{\bar{U}} \phi d f_{\infty}
$$

for any $\phi \in C(\bar{U})$. 
Lemma 4.1. Up to a subsequence, the measures $f_{p}$ converge weakly in measure in $\bar{U}$ to some measure $f_{\infty}$ supported in $\bar{U}$ satisfying

$$
f_{\infty}(\bar{U})=0 \quad \text { and } \quad\left|f_{\infty}\right|(\bar{U})=1 .
$$

Proof. We claim that

$$
\lim _{p \rightarrow+\infty} \int_{U}\left|f_{p}\right| d x=1 .
$$

First, in view of (4.1), we have that

$$
\int_{U}\left|f_{p}\right| d x \leq\left\|f_{p}\right\|_{L^{p^{\prime}(U)}}|U|^{1-1 / p^{\prime}}=|U|^{1-1 / p^{\prime}} \rightarrow 1 \quad \text { as } p \rightarrow+\infty
$$

and then, recalling that $u_{p} \rightarrow u$ in $C(\bar{U})$ with $\|u\|_{L^{\infty}(U)}=1$,

$$
1=\int_{U} u_{p} f_{p} d x \leq\left\|u_{p}\right\|_{L^{\infty}(U)}\left\|f_{p}\right\|_{L^{1}(U)}=(1+o(1))\left\|f_{p}\right\|_{L^{1}(U)} .
$$

It follows in particular that the measures $\left|f_{p}\right|$ are bounded in $M(\bar{U})$ independently of $p$. Since $\bar{U}$ is compact, we can then extract from this sequence a subsequence converging weakly to some measure $f_{\infty} \in M(\bar{U})$. Passing to the limit in (4.1) and (4.3) gives (4.2).

Consider the functionals $G_{p}, G_{\infty}: C(\bar{U}) \times M(\bar{U}) \rightarrow \mathbb{R} \cup\{+\infty\}$ defined by

$$
G_{p}(v, \sigma)= \begin{cases}-\int_{U} v \sigma d x & \text { if } \sigma \in L^{p^{\prime}}(U),\|\sigma\|_{L^{p^{\prime}}(U)} \leq 1, \int_{U} \sigma d x=0, \\ & \text { and } v \in W^{s, p}(U), \llbracket v \rrbracket_{s, p} \leq\left(\lambda_{p, s}^{N}\right)^{1 / p}, \\ +\infty & \text { otherwise, }\end{cases}
$$

and

$$
G_{\infty}(v, \sigma)= \begin{cases}-\int_{U} v d \sigma, & \text { if } \sigma \in M(\bar{U}),|\sigma|(\bar{U}) \leq 1, \sigma(\bar{U})=0, \\ & \text { and } v \in W^{s, \infty}(U),|v(x)-v(y)| \leq \Lambda_{\infty, s}^{N} d(x, y)^{s} \\ +\infty & \text { otherwise. }\end{cases}
$$

Remark that these functionals are similar to the ones considered for the Dirichlet case but the spaces involved change. In fact, here we consider $W^{s, p}(U)$ instead of $\widetilde{W}^{s, p}(U)$ (that encodes the fact that we are considering functions that vanish outside $U$ when dealing with the Dirichlet problem).

As for the Dirichlet case, we can prove as in $[5,19]$ that $G_{\infty}$ is the limit of the $G_{p}$ in the sense of $\Gamma$-convergence:

Lemma 4.2. The functionals $G_{p}$ converge in the sense of $\Gamma$-convergence to $G_{\infty}$

The proof is similar as that of Proposition 3.7 in [5] and hence we omit it. As a corollary we obtain that 
Lemma 4.3. Let $u_{p}$ be an extremal for $\lambda_{p, s}^{N}$, then $\left(u_{p}, f_{p}\right)$ is a minimizer for $G_{p}$, and any limit $\left(u_{\infty}, f_{\infty}\right)$ along a subsequence $p_{j} \rightarrow+\infty$ is a minimizer for $G_{\infty}$, with

$$
G_{\infty}\left(u_{\infty}, f_{\infty}\right)=\lim _{p \rightarrow+\infty} G_{p}\left(u_{p}, f_{p}\right)=-1 .
$$

Proof. Notice that the pair $\left(u_{p}, f_{p}\right)$ is a minimizer of $G_{p}$. Indeed, given a pair $(v, \sigma)$ admissible for $G_{p}$ take $\bar{v} \in \mathbb{R}$ such that

$$
\int_{U}|v-\bar{v}|^{p-2}(v-\bar{v}) d x=0
$$

Then, recalling that $\int_{U} \sigma d x=0$ and the definition of $\lambda_{p, s}^{N}$, we have

$$
\begin{aligned}
G_{p}(v, \sigma) & =-\int_{U}(v-\bar{v}) \sigma d x \\
& \geq-\|v-\bar{v}\|_{L^{p}(U)}\|\sigma\|_{L^{p^{\prime}}(U)} \\
& \geq-\left(\lambda_{p, s}^{N}\right)^{-1 / p} \llbracket v-\bar{v} \rrbracket_{s, p} \\
& \geq-1=G_{p}\left(u_{p}, f_{p}\right)
\end{aligned}
$$

Moreover $\left(u_{p}, f_{p}\right) \rightarrow\left(u_{\infty}, f_{\infty}\right)$ along a sequence $p_{j} \rightarrow+\infty$. Then, it follows that

$$
\liminf _{p \rightarrow+\infty}\left(\inf G_{p}\right)=\liminf _{p \rightarrow+\infty} G_{p}\left(u_{p}, f_{p}\right) \geq G_{\infty}\left(u_{\infty}, f_{\infty}\right) \geq \inf _{B} G_{\infty}
$$

where $B$ is the set of all pairs $(v, \sigma) \in W^{s, \infty}(U) \times M(\bar{U})$ such that

$$
|\sigma|(\bar{U}) \leq 1, \sigma(\bar{U})=0, \text { and }|v(x)-v(y)| \leq \Lambda_{\infty, s}^{N} d(x, y)^{s}
$$

Moreover, the limsup property implies that

$$
\limsup _{p \rightarrow+\infty}\left(\inf _{B} G_{p}\right) \leq \inf _{B} G_{\infty} .
$$

Hence

$$
\lim _{p \rightarrow+\infty} \inf _{B} G_{p}=\lim _{p \rightarrow \infty} G_{p}\left(u_{p}, f_{p}\right)=G_{\infty}\left(u_{\infty}, f_{\infty}\right)=\inf _{B} G_{\infty}
$$

We can now relate $\Lambda_{s, \infty}^{N}$ to $W_{s}$. Recall that if $\sigma \in M(\bar{U})$, then $\sigma^{ \pm} \in M(\bar{U})$ denote the positive and negative part of $\sigma$. In particular, $\sigma=\sigma^{+}-\sigma^{-}$, and $|\sigma|=\sigma^{+}+\sigma^{-}$.

Proof of Theorem 1.2. The conditions $\sigma(\bar{U})=0$ and $|\sigma|(\bar{U})=1$ are equivalent to

$$
\sigma^{+}(\bar{U})=\sigma^{-}(\bar{U})=1 / 2 .
$$

We can therefore rewrite the fact that the pair $\left(u_{\infty}, f_{\infty}\right)$ is a minimizer of $G_{\infty}$ as

$$
1=\max _{\sigma \in M_{1 / 2}} \max _{v \in F_{\Lambda_{s, \infty}^{N}}} \int_{U} v d\left(\sigma^{+}-\sigma^{-}\right),
$$


where

$$
M_{t}=\left\{\sigma \in M(\bar{U}): \sigma^{+}(U)=\sigma^{+}(U)=t\right\},
$$

and

$$
F_{R}=\left\{v \in W^{s, \infty}(U):|v(x)-v(y)| \leq R d(x, y)\right\}
$$

that is,

$$
\frac{2}{\Lambda_{\infty, s}^{N}}=\max _{\sigma \in M_{1}} \max _{v \in F_{1}} \int_{U} v d\left(\sigma^{+}-\sigma^{-}\right)
$$

Then, we obtain the conclusion (1.3), recalling the definition of $W_{s}$ given by (1.5).

\section{Eigenvalue problems With a Different Neumann boundary CONDITION}

In this section we prove Theorem 1.3. For this purpose, first we present some previous results.

Theorem 5.1. The spaces

$$
\mathcal{W}^{s, p}(U):=\left\{u: \mathbb{R}^{n} \rightarrow \mathbb{R} \text { measurable }:\|u\|_{L^{p}(U)}^{p}+\mathcal{H}_{s, p}(u)<+\infty\right\}
$$

and

$$
\mathcal{W}^{s, \infty}(U):=\left\{u: \mathbb{R}^{n} \rightarrow \mathbb{R} \text { measurable }:\|u\|_{L^{\infty}(U)}+\mathcal{H}_{s, \infty}(u)<+\infty\right\}
$$

are Banach spaces with the norms

$$
\|u\|_{\mathcal{W}^{s, p}(U)}^{p}:=\|u\|_{L^{p}(U)}^{p}+\mathcal{H}_{s, p}(u)
$$

and

$$
\|u\|_{\mathcal{W}^{s, \infty}(U)}:=\|u\|_{L^{\infty}(U)}+\mathcal{H}_{s, \infty}(u)
$$

respectively.

The proof follows exactly as in the proof of [9, Proposition 3.1].

Remark 5.2. It holds that $\mathcal{W}^{s, p}(U) \subset W^{s, p}(U)$.

Remark 5.3. The operator $I: \mathcal{W}^{s, p}(U) \rightarrow E=L^{p}(U) \times L^{p}\left(\mathbb{R}^{2 n} \backslash\left(U^{c}\right)^{2}\right)$ given by

$$
I(u):=\left(u, \frac{u(x)-u(y)}{d(x, y)^{\frac{n}{p}+s}}\right)
$$

is an isometry. Then $I\left(\mathcal{W}^{s, p}(U)\right)$ is a closed subspace of $E$ due to the fact that $\mathcal{W}^{s, p}(U)$ is a Banach space. Hence $I\left(\mathcal{W}^{s, p}(U)\right)$ is reflexive since $E$ is reflexive. Then, $\mathcal{W}^{s, p}(U)$ is reflexive.

Following the proofs of Lemmas 3.2 and 3.7 in [9], we have the following result. 
Lemma 5.4. Let $u$ and $v$ be bounded $C^{2}$ functions in $\mathbb{R}^{n}$. Then the following formulae hold:

Divergence theorem

$$
\int_{U}(-\Delta)_{p}^{s} u(x) d x=-\int_{\mathbb{R} \backslash U} \mathcal{N}_{s, p} u(x) d x .
$$

Integration by parts formula

$$
\frac{1}{2} \mathcal{H}_{s, p}(u, v)=\int_{U} v(x)(-\Delta)_{p}^{s} u(x) d x+\int_{\mathbb{R} \backslash U} v(x) \mathcal{N}_{s, p} u(x) d x,
$$

where

$\mathcal{H}_{s, p}(u, v):=\iint_{\mathbb{R}^{2 n} \backslash\left(U^{c}\right)^{2}} \frac{|u(x)-u(y)|^{p-2}(u(x)-u(y))(v(x)-v(y))}{d(x, y)^{n+p s}} d x d y$.

This result leads us to the following definition.

Definition 5.5. A function $u \in \mathcal{W}^{s, p}(U)$ is a weak solution of (1.4) if

$$
\frac{1}{2} \mathcal{H}_{s, p}(u, v)=\lambda \int_{U}|u|^{p-2} u v d x
$$

for all $v \in \mathcal{W}^{s, p}(U)$.

In this context we have the following definition.

Definition 5.6. We say that $\lambda$ is a fractional Neumann $p$-eigenvalue provided there exists a nontrivial weak solution $u \in \mathcal{W}^{s, p}(U)$ of (1.4). The function $u$ is a corresponding eigenfunction.

Let us observe the following: if $\lambda>0$ is an eigenvalue and $u$ is an eigenfunction associated to $\lambda$, then, taking $v \equiv 1$ as a test function in (5.1), we have

$$
\int_{U}|u|^{p-2} u d x=0
$$

In fact, we have that $\lambda=0$ is the first eigenvalue of our problem.

Lemma 5.7. It holds that $\lambda=0$ is an eigenvalue of (1.4) (with $u=1$ as eigenfunction), and it is isolated and simple.

Proof. Let $u$ be an eigenfunction corresponding to $\lambda=0$ in problem (1.4). From (5.1) taking $v=u$ as a test function we obtain that $u$ is constant in $U$.

Now, if we have a sequence of eigenvalues $\lambda_{k} \rightarrow 0$ then the corresponding eigenfunctions, $u_{k}$, normalized by $\left\|u_{k}\right\|_{L^{p}(U)}=1$, converge to some $u$. It is not difficult to show that $u$ is an eigenfunction corresponding to $\lambda=0$ (consequently, $u \equiv$ const) with $\|u\|_{L^{p}(U)}=1$ and $\int_{U}|u|^{p-2} u d x=0$, a contradiction that shows that $\lambda=0$ is an isolated eigenvalue. 
Thus, the existence of the first non-zero eigenvalue of (1.4) is related to the problem of minimizing the following non-local quotient

$$
\frac{\mathcal{H}_{s, p}(v)}{2\|v\|_{L^{p}(U)}^{p}}
$$

among all functions $v \in \mathcal{W}^{s, p}(U) \backslash\{0\}$ such that $\int_{U}|v|^{p-2} v d x=0$.

We are now ready to prove Theorem 1.3. For simplicity, we divide the proof of this theorem into three parts contained in the following lemmas.

First, by a standard compactness argument and using that $\mathcal{W}^{s, p}(U) \subset$ $W^{s, p}(U)$, we have that $\lambda_{s, p}$ is the first non-zero eigenvalue of (1.4).

Lemma 5.8. It holds that $\lambda_{s, p}$ is the first non-zero eigenvalue of (1.4).

Remark 5.9. Since $\mathcal{W}^{s, p}(U) \subset W^{s, p}(U)$ and

$$
\llbracket u \rrbracket_{s, p}^{p} \leq \mathcal{H}_{s, p}(u), \quad \forall u \in \mathcal{W}^{s, p}(U)
$$

we have that

$$
\lambda_{s, p}^{N} \leq 2 \lambda_{s, p}
$$

Our next result shows the asymptotic behavior of $\left(\lambda_{s, p}\right)^{1 / p}$.

Lemma 5.10. We have

$$
\lim _{p \rightarrow+\infty}\left(\lambda_{s, p}\right)^{1 / p}=\frac{2}{\left(\operatorname{diam}_{d}(U)\right)^{s}}=\Lambda_{s, \infty}:=\inf \left\{\frac{\mathcal{H}_{s, \infty}(u)}{\|u\|_{L^{\infty}(U)}}: u \in \mathcal{A}\right\},
$$

where

$$
\mathcal{A}:=\left\{v \in \mathcal{W}^{s, \infty}(U) \backslash\{0\}: \sup _{x \in U} u(x)+\inf _{x \in U} u(x)=0\right\} .
$$

Proof. For the reader's convenience, we split the proof in four steps.

Step 1. We start showing that

$$
\Lambda_{s, \infty} \leq \frac{2}{\left(\operatorname{diam}_{d}(U)\right)^{s}} .
$$

Let $x_{0}, y_{0} \in \bar{U}$ such that $d\left(x_{0}, y_{0}\right)=\operatorname{diam}_{d}(U)$. Let $u: \mathbb{R}^{n} \rightarrow \mathbb{R}$ be given by

$$
u(x):=-1+\frac{2}{\operatorname{diam}_{d}(U)} d\left(x, y_{0}\right)^{s} .
$$

Observe that,

$$
\sup _{x \in U} u(x)=-\inf _{x \in U} u(x)=1
$$

and

$$
\frac{|u(x)-u(y)|}{d(x, y)^{s}}=\frac{2}{\left(\operatorname{diam}_{d}(U)\right)^{s}} \frac{\left|d\left(x, y_{0}\right)^{s}-d\left(y, y_{0}\right)^{s}\right|}{d(x, y)^{s}} \leq \frac{2}{\left(\operatorname{diam}_{d}(U)\right)^{s}}
$$

for all $x, y \in \mathbb{R}^{n}$. Then $u \in \mathcal{A},\|u\|_{L^{\infty}(U)}=1$ and

$$
\mathcal{H}_{s, \infty}(u) \leq \frac{2}{\left(\operatorname{diam}_{d}(U)\right)^{s}}
$$


Therefore

$$
\Lambda_{s, \infty} \leq \mathcal{H}_{s, \infty}(u) \leq \frac{2}{\left(\operatorname{diam}_{d}(U)\right)^{s}}
$$

Step 2. We now prove that

$$
\Lambda_{s, \infty} \geq \frac{2}{\left(\operatorname{diam}_{d}(U)\right)^{s}}
$$

If $u \in \mathcal{A}$ then

$$
\begin{aligned}
2\|u\|_{L^{\infty}(U)} & =\sup _{x \in U} u(x)-\inf _{x \in U} u(x) \\
& =\sup \{|u(x)-u(y)|: x, y \in U\} \\
& \leq\left(\operatorname{diam}_{d}(U)\right)^{s} \sup \left\{\frac{|u(x)-u(y)|}{d(x, y)^{s}}: x, y \in U\right\} \\
& \leq\left(\operatorname{diam}_{d}(U)\right)^{s} \mathcal{H}_{s, \infty}(u) .
\end{aligned}
$$

Thus

$$
\frac{2}{\left(\operatorname{diam}_{d}(U)\right)^{s}} \leq \frac{\mathcal{H}_{s, \infty}(u)}{\|u\|_{L^{\infty}(U)}}
$$

for any $u \in \mathcal{A}$, that is,

$$
\Lambda_{s, \infty} \geq \frac{2}{\left(\operatorname{diam}_{d}(U)\right)^{s}}
$$

Step 3. We show that

$$
\frac{2}{\left(\operatorname{diam}_{d}(U)\right)^{s}} \leq \liminf _{p \rightarrow+\infty}\left(\lambda_{s, p}\right)^{1 / p}
$$

By (1.2) and Remark 5.9, we have that

$$
\begin{aligned}
\frac{2}{\left(\operatorname{diam}_{d}(U)^{s}\right)} & \leq \lim _{p \rightarrow+\infty}\left(\lambda_{s, p}^{N}\right)^{1 / p} \\
& \leq \liminf _{p \rightarrow+\infty} 2^{1 / p}\left(\lambda_{s, p}(U)\right)^{1 / p}=\liminf _{p \rightarrow+\infty}\left(\lambda_{s, p}(U)\right)^{1 / p} .
\end{aligned}
$$

Step 4. Finally, we prove that

$$
\limsup _{p \rightarrow+\infty}\left(\lambda_{s, p}\right)^{1 / p} \leq \frac{2}{\left(\operatorname{diam}_{d}(U)\right)^{s}} .
$$

As in Step 1, let $x_{0}, y_{0} \in \bar{U}$ be such that $d\left(x_{0}, y_{0}\right)=\operatorname{diam}_{d}(U)$. Set $\delta=$ $\operatorname{diam}_{d}(U)$,

$$
U_{\delta}:=\left\{x \in \mathbb{R}^{n}: \inf _{y \in U} d(x, y) \leq \delta\right\}
$$

and

$$
u(x):= \begin{cases}d\left(x, y_{0}\right) & \text { if } x \in U_{\delta} \\ 0 & \text { if } x \in \mathbb{R}^{n} \backslash U_{\delta}\end{cases}
$$


Let $\varepsilon>0$. Then

$$
\begin{aligned}
\mathcal{H}_{s, p}(u) \leq 2 \int_{U \times U_{\delta}} & \frac{\left|d\left(x, y_{0}\right)-d\left(y, y_{0}\right)\right|^{p}}{d(x, y)^{n+s p}} d x d y \\
& +2 \int_{U \times\left(\mathbb{R}^{n} \backslash U_{\delta}\right)} \frac{d\left(x, y_{0}\right)^{p}}{d(x, y)^{n+s p}} d x d y \\
\leq 2 \int_{U \times U_{\delta}} & \frac{d(x, y)^{p(1-s)-\varepsilon}}{d(x, y)^{n-\varepsilon}} d x d y \\
& +2 \int_{U \times\left(\mathbb{R}^{n} \backslash U_{\delta}\right)} \frac{d\left(x, y_{0}\right)^{p}}{d(x, y)^{n+\varepsilon+s p-\varepsilon}} d x d y .
\end{aligned}
$$

Thus, since $d$ is a distance equivalent to the Euclidean one, if

$$
p>\max \left\{\frac{\varepsilon}{(1-s)}, \frac{\varepsilon}{s}\right\}
$$

we get that $u \in \mathcal{W}^{s, p}(U)$ and

$$
\mathcal{H}_{s, p}(u) \leq C\left(\operatorname{diam}_{d}(U)\right)^{p(1-s)}\left\{\left(\operatorname{diam}_{d}(U)\right)^{-\varepsilon}+\left(\operatorname{diam}_{d}(U)\right)^{\varepsilon}\right\},
$$

where $C$ is a constant independent of $p$.

We now choose $c_{p} \in \mathbb{R}$ such that

$$
w_{p}(x)=u(x)-c_{p}
$$

satisfies

$$
\int_{U}\left|w_{p}\right|^{p-2} w_{p} d x=0
$$

Hence, if $p>\max \{\varepsilon /(1-s), \varepsilon / s\}$, by (5.2), we have that

$$
\begin{aligned}
\lambda_{s, p} & \leq \frac{\mathcal{H}\left(w_{p}\right)}{2\left\|w_{p}\right\|_{L^{p}(U)}^{p}} \\
& =\frac{\mathcal{H}(u)}{2\left\|w_{p}\right\|_{L^{p}(U)}^{p}} \\
& \leq \frac{C}{2\left\|w_{p}\right\|_{L^{p}(U)}^{p}}\left(\operatorname{diam}_{d}(U)\right)^{p(1-s)}\left\{\left(\operatorname{diam}_{d}(U)\right)^{-\varepsilon}+\left(\operatorname{diam}_{d}(U)\right)^{\varepsilon}\right\},
\end{aligned}
$$

therefore

$$
\limsup _{p \rightarrow+\infty}\left(\lambda_{s, p}\right)^{1 / p} \leq \frac{\left(\operatorname{diam}_{d}(U)\right)^{1-s}}{\liminf _{p \rightarrow+\infty}\left\|w_{p}\right\|_{L^{p}(U)}} .
$$

On the other hand, in [10] it is proved that

$$
\liminf _{p \rightarrow+\infty}\left\|w_{p}\right\|_{L^{p}(U)} \geq \frac{2}{\operatorname{diam}_{d}(U)} .
$$

Thus, by (5.3) and (5.4), we get

$$
\limsup _{p \rightarrow+\infty}\left(\lambda_{s, p}\right)^{1 / p} \leq \frac{2}{\left(\operatorname{diam}_{d}(U)\right)^{s}} .
$$


This concludes the proof.

Remark 5.11. By (1.2) and Lemma 5.10, we have that

$$
\Lambda_{s, \infty}^{N}=\lim _{p \rightarrow+\infty}\left(\lambda_{s, p}^{N}\right)^{1 / p}=\frac{2}{\left(\operatorname{diam}_{d}(U)\right)^{s}}=\lim _{p \rightarrow+\infty}\left(\lambda_{s, p}\right)^{1 / p}=\Lambda_{s, \infty} .
$$

Concerning the convergence as $p \rightarrow+\infty$ of the eigenfunctions we have the following result.

Lemma 5.12. If $u_{p}$ is a minimizer of $\lambda_{s, p}$, normalized with $\left\|u_{p}\right\|_{L^{p}(U)}=1$, then, up to a subsequence, $u_{p}$ converges in $C(\bar{U})$ to some minimizer $u_{\infty} \in$ $W^{s, \infty}(U)$ of $\Lambda_{s, \infty}^{N}$.

Proof. For any $p \in(1, \infty)$, we consider $u_{p} \in \mathcal{W}^{s, p}(U)$ such that

$$
\left\|u_{p}\right\|_{L^{p}(U)}=1, \quad \int_{U}\left|u_{p}\right|^{p-2} u_{p} d x=0 \quad \text { and } \quad \frac{1}{2} \mathcal{H}_{s, p}\left(u_{p}\right)=\lambda_{s, p} .
$$

Then, by Lemma 5.10, there exists a constant $C$ independent of $p$ such that

$$
\left(\frac{\mathcal{H}_{s, p}\left(u_{p}\right)}{2}\right)^{\frac{1}{p}} \leq C
$$

for all $p \in(1, \infty)$.

Let us fix $q \in(1, \infty)$ such that $s q>2 n$. If $p>q$ then, by Hölder's inequality, we have that

$$
\left\|u_{p}\right\|_{L^{q}(\Omega)} \leq|U|^{\frac{1}{q}-\frac{1}{p}}\left\|u_{p}\right\|_{L^{p}(\Omega)} \leq|U|^{\frac{1}{q}-\frac{1}{p}} \quad \forall p \geq q,
$$

and taking $r=s-n / q \in(0,1)$, again by Hölder's inequality, we get

$$
\begin{aligned}
\llbracket u_{p} \rrbracket_{r, q}^{q} & =\int_{U} \int_{U} \frac{\left|u_{p}(x)-u_{p}(y)\right|^{q}}{d(x, y)^{s q}} d x d y \\
& \leq|U|^{2\left(1-\frac{q}{p}\right)}\left(\int_{U} \int_{U} \frac{\left|u_{p}(x)-u_{p}(y)\right|^{p}}{d(x, y)^{s p}} d x d y\right)^{\frac{q}{p}} \\
& \leq 2^{\frac{q}{p}}\left(\operatorname{diam}_{d}(U)\right)^{\frac{n q}{p}}|U|^{2\left(1-\frac{q}{p}\right)}\left(\frac{\mathcal{H}_{s, p}\left(u_{p}\right)}{2}\right)^{\frac{q}{p}} .
\end{aligned}
$$

Then, by (5.5), we get

$$
\llbracket u_{p} \rrbracket_{r, q} \leq 2^{\frac{1}{p}}\left(\operatorname{diam}_{d}(U)\right)^{\frac{n}{p}}|U|^{2\left(\frac{1}{q}-\frac{1}{p}\right)} C^{q} \quad \forall p \geq q,
$$

where $C$ is a constant independent of $p$. Hence $\left\{u_{p}\right\}_{p \geq q}$ is a bounded sequence in $W^{r, q}(U)$. Then, since $r q=s q-n>n$, by fractional compact embedding theorems (see [11, Theorem 4.54]), there exist a function $u_{\infty} \in C(\bar{U})$ and a subsequence $\left\{u_{p_{j}}\right\}_{j \in \mathbb{N}}$ of $\left\{u_{p}\right\}_{p \geq q}$, such that

$$
\begin{array}{ll}
u_{p_{j}} \rightarrow u_{\infty} & \text { uniformly in } \bar{U}, \\
u_{p_{j}} \rightarrow u_{\infty} & \text { weakly in } W^{r, q}(U) .
\end{array}
$$


Hence, by (5.6), $\left\|u_{\infty}\right\|_{L^{q}(\Omega)} \leq|U|^{\frac{1}{q}}$, and by (5.7) and Remark 5.11, we get

$$
\begin{aligned}
\llbracket u_{\infty} \rrbracket_{r, q} & \leq \liminf _{j \rightarrow \infty} \llbracket u_{p_{j}} \rrbracket_{r, q} \\
& \left.\leq \liminf _{j \rightarrow \infty} 2^{1 / p_{j}}\left(\operatorname{diam}_{d}(U)\right)^{n / p_{j}}|U|^{2\left(1-1 / p_{j}\right.}\right)\left(\frac{\mathcal{H}_{s, p_{j}}\left(u_{p_{j}}\right)}{2}\right)^{1 / p_{j}} \\
& =|U|^{\frac{2}{q}} \Lambda_{s, \infty}^{N} .
\end{aligned}
$$

Letting $q \rightarrow+\infty$, we obtain

$$
\left\|u_{\infty}\right\|_{L^{\infty}(\Omega)} \leq 1
$$

and

$$
\llbracket u_{\infty} \rrbracket_{s, \infty} \leq \Lambda_{s, \infty}^{N}
$$

On the other hand,

$$
1=\left\|u_{p_{j}}\right\|_{L^{p_{j}}(U)} \leq|U|^{\frac{1}{p_{j}}}\left\|u_{p_{j}}\right\|_{L^{\infty}(U)} \quad \forall j \in \mathbb{N},
$$

then

Hence

$$
1 \leq\left\|u_{\infty}\right\|_{L^{\infty}(U)}
$$

and by (5.8) we get

$$
\left\|u_{\infty}\right\|_{L^{\infty}(U)}=1
$$

$$
\frac{\llbracket u_{\infty} \rrbracket_{s, \infty}}{\left\|u_{\infty}\right\|_{L^{\infty}(U)}} \leq \Lambda_{s, \infty}^{N}
$$

Finally, in $[10,19]$ it was proved that the condition

$$
\int_{U}\left|u_{p_{j}}\right|^{p_{j}-2} u_{p_{j}} d x=0
$$

leads to

$$
\sup _{x \in U} u_{\infty}(x)+\inf _{x \in U} u_{\infty}(x)=0
$$

in the limit as $p \rightarrow+\infty$. Then, using (5.9), we have that $u_{\infty}$ is a minimizer of $\Lambda_{s, \infty}^{N}$.

\section{REFERENCES}

[1] G. Aronsson, Extensions of functions satisfying Lipschitz conditions. Ark. Math., 6 (1967), 551-561.

[2] G. Aronsson, M.G. Crandall and P. Juutinen, A tour of the theory of absolutely minimizing functions. Bull. Amer. Math. Soc., 41 (2004), 439-505.

[3] M. Belloni and B. Kawohl, The pseudo-p-Laplace eigenvalue problem and viscosity solutions as $p \rightarrow \infty$. ESAIM Control Optim. Calc. Var., 10 (2004), 28-52.

[4] T. Bhattacharya, E. Di Benedetto and J. Manfredi, Limits as $p \rightarrow \infty$ of $\Delta_{p} u_{p}=f$ and related extremal problems. Rend. Sem. Mat. Univ. Politec. Torino, (1991), 15-68.

[5] T. Champion, L. De Pascale, C. Jimenez, The $\infty$ - eigenvalue problem and a problem of optimal transportation. Commim. Appl. Anal., 13 (4), (2009), 547-565. 
[6] M.G. Crandall, H. Ishii and P.L. Lions. User's guide to viscosity solutions of second order partial differential equations. Bull. Amer. Math. Soc., 27 (1992), 1-67.

[7] G. Dal Maso. An introduction to G-convergence. Progress in Nonlinear Differential Equations and their Applications, 8. Birkhuser Boston, Inc., Boston, MA, 1993.

[8] L. M. Del Pezzo and A. Salort. The first non-zero Neumann p-fractional eigenvalue, (2014), eprint arXiv:1409.0840.

[9] S. Dipierro, X. Ros-Oton, and E. Valdinoci, Nonlocal problems with Neumann boundary conditions, (2014), eprint arXiv:1407.3313.

[10] L. Esposito, B. Kawohl, C. Nitsch, and C. Trombetti, The Neumann eigenvalue problem for the $\infty$-Laplacian, (2014), eprint arXiv:1405.3535.

[11] F. Demengel and G. Demengel, Functional spaces for the theory of elliptic partial differential equations, Universitext, Springer, London, 2012, Translated from the 2007 French original by Reinie Erné.

[12] J. Garcia-Azorero, J.J. Manfredi, I. Peral, J.D. Rossi. Steklov eigenvlue for the $\infty$ Laplacian. Rendiconti Lincei, 17 (3), (2006), 199-210.

[13] R. Jensen, Uniqueness of Lipschitz extensions: minimizing the sup norm of the gradient. Arch. Rational Mech. Anal., 123, (1993), 51-74.

[14] H. Jylha, An optimal transportation problem related to the limits of solutions of local and nonlocal p-Laplace-type problems. Rev. Mat. Complutense, 28(1), (2015), 85121.

[15] P. Juutinen, P. Lindqvist and J. J. Manfredi, The $\infty$-eigenvalue problem. Arch. Rational Mech. Anal., 148, (1999), 89-105.

[16] P. Juutinen and P. Lindqvist, On the higher eigenvalues for the $\infty$-eigenvalue problem. Calc. Var. Partial Differential Equations, 23, (2005), no. 2, 169-192.

[17] A. Lê, On the first eigenvalue of the Steklov eigenvalue problem for the infinity Laplacian, Electronic Journal of Differential Equations, 2006, (111), 1-9.

[18] E. Lindgren and P. Lindqvist, Fractional eigenvalues. Calc. Var. Partial Differential Equations, 49, (2014), no. 1-2, 795-826.

[19] J. D. Rossi, N. Saintier. On the first nontrivial eigenvalue of the $\infty$-Laplacian with Neumann boundary conditions. To appear in Houston Journal of Mathematics.

[20] C. Villani, Optimal transport, old and new, Grundlehren der Mathematischen Wissenschaften, 338. Springer-Verlag, Berlin, 2009.

(L. M. Del Pezzo, J. D. Rossi, N. Saintier, A. Salort)

COnicet and Dep. de Matemática, FCEyn, Universidad de Buenos Aires, Ciudad Universitaria, Pab 1 (1428), Buenos Aires, Argentina.

E-mail address:

ldpezzo@dm.uba.ar

jrossi@dm.uba.ar

nsaintier@dm.uba.ar

asalort@dm.uba.ar

$U R L$ :

http://cms.dm.uba.ar/Members/ldpezzo

http://mate.dm.uba.ar/ jrossi/ 